\title{
Commercial Polycarbonate Track-Etched Membranes as Optical Chemical Sensors ${ }^{\dagger}$
}

\author{
Paula Martínez-Pérez and Jaime García-Rupérez * \\ Nanophotonics Technology Center, Universitat Politècnica de València, Camino de Vera s/n, \\ 46022 Valencia, Spain; paumarp3@ntc.upv.es \\ * Correspondence: jaigarru@ntc.upv.es; Tel.: +34-963-879-736 \\ + Presented at the 5th International Electronic Conference on Sensors and Applications, 15-30 November \\ 2018; Available online: https://ecsa-5.sciforum.net.
}

Published: 14 November 2018

\begin{abstract}
Refractive index is the main parameter measured by current optical sensors. Among all the photonic structures available for their design, porous materials have become an excellent option, since they provide better sensitivities. In our work, commercially available polycarbonate tracketched membranes were used as porous photonic structures. By means of reflectivity measurements, we demonstrated their capability to detect the presence of ethanol in the medium and showed the possibility of reusing them in several sensing assays. This new material could become an easier-to-obtain and cheaper alternative to current porous materials commonly used in optical sensors for refractive index sensing.
\end{abstract}

Keywords: optical sensor; polycarbonate track-etched membrane; refractive index sensing; silicon

\section{Introduction}

Sensors are devices which are widely used in our daily life as they can be designed to detect physical, chemical, or biological agents of interest in areas as diverse as industrial procedures, security, and medical diagnosis or defense, among others. According to the transducer element, they can be classified as mechanical, optical, thermal, electromagnetic, or electrochemical. Amongst these, optical sensors have gained most interest in recent years due to their high sensitivity and specificity, and real-time, direct, and label-free detection and multiplexing capabilities, miniaturizing possibilities, cost-effectiveness, and immunity to electromagnetic interferences [1].

There are different kinds of optical sensors, but those based on the measurement of changes of the refractive index (RI) are widely used as they allow the optical label-free detection of nonionic, transparent in the UV/vis range, or non-fluorescent molecules [2]. For their design, different photonic structures have been developed. Most of them are based on the interaction of the evanescent optical field with the analyte [3]. However, the achievable sensitivity values of these sorts of structures are low, as only a low-intensity region of the optical field interacts with the analyte in a small surface. This issue can be overcome by using porous structures, which allow the whole optical field to interact with the analyte, since the recognition occurs inside the light propagation medium. Moreover, the presence of pores on its surface increases the surface-to-volume ratio, which allows for the adhesion of more receptors and hence, the detection of more analytes. Consequently, this favors the interaction of light with the analyte and leads to a sensitivity increase as well [4].

Porous silicon (PSi) is the most commonly used porous photonic structure as it is easy, cheap, and fast to fabricate. Furthermore, its surface chemistry has long been studied, thus its functionalization to create specific sensors for specific applications in different fields is relatively easy [5]. 
Besides PSi, several research groups are also working on other materials, such as polymers [6] or metals [7] for the development of such structures.

The aim of this work was to find an alternative porous material to create porous photonic structures. For such a purpose, we employed commercially available polycarbonate track-etched (PCTE) membranes. These membranes are porous layers that is reminiscent of the structure of a Fabry-Pérot (FP) interferometer typically made on PSi. These photonic structures consist of a monolayer of a porous material, and have been long used for chemical and biochemical sensing [8]; thus, we hypothesized the utility of PCTE membranes for such an aim as well.

To assess it, we studied the optical behavior in the near-infrared (NIR) region of PCTE membranes, optimized it by placing the membrane on a flat, polished silicon surface, and then performed reflectivity measurements in the presence of two different concentrations of ethanol. What we observed was a spectral shift that confirmed our hypothesis about the utility of commercial membranes for optical sensing. Furthermore, we could observe differences depending on the ethanol concentration they were exposed to. This could indicate the suitability of these kinds of structures to perform quantitative assays, but further assays are needed to confirm it. Finally, we also saw that these sensors can be used more than once, allowing us to do continuous measurement cycles.

\section{Materials and Methods}

PCTE membranes (19 $\mathrm{mm}$ diameter and $30 \mathrm{~nm}$ pore diameter) were purchased from Whatman. Firstly, the surface of these commercial membranes were activated by oxygen plasma in a plasma asher (PVA TEPLA 200, PVA TePla AG, Wettenberg, Germany) for $1 \mathrm{~min}$ (50 W, $1.5 \mathrm{mBar}$ ). Immediately, the samples were immersed in an aqueous solution of 5\% 3-amino-propyltriethoxysilane (APTES) (SigmaAldrich, St Louis, MO, USA) at $80^{\circ} \mathrm{C}$ for $20 \mathrm{~min}$. After that, the samples were removed from the solution and dried out in a cleanroom wipe. Once dried, they could be placed on a previously activated silicon wafer surface by piranha treatment $\left(\mathrm{H}_{2} \mathrm{SO}_{4}: \mathrm{H}_{2} \mathrm{O}=3: 1\right)$ for $10 \mathrm{~min}$.

For measuring the refractive index changes caused by the presence of ethanol (Scharlab, Barcelona, Spain), a Bruker FTIR system (Bruker Corporation, Billerica, MA, USA) was employed. The reflectivity measurements were performed in the NIR range $(1110-2500 \mathrm{~nm})$, with a resolution of $4 \mathrm{~cm}^{-1}$. To enhance the $\mathrm{S} / \mathrm{N}$ ratio, 30 scans were collected every minute to do a continuous monitoring of the spectrum shift evolution. For the graphical representation of the spectra, MATLAB R2016b (The MathWorks, Inc., Natick, MA, USA) was used.

In order to characterize the morphology of the surface of PCTE membranes, a Field-Emission Scanning Electron Microscope (FESEM) Hitachi S-4500 (Hitachi, Ltd., Chiyoda, Japan) was employed.

\section{Results}

\subsection{Silicon Substrate to Enhance the Optical Response of PCTE Membranes}

The PCTE membranes are porous monolayers that is reminiscent of the structure of FP interferometers (Figure 1). For this reason, we hypothesized that PCTE membranes would have the same optical response as FP interferometers - an interference fringe pattern-which is the result of the interferences generated by the multiple reflections experimented by light while travelling through the different interfaces generated by the porous structure and its surroundings.

Every porous monolayer, when illuminated, generates a reflectivity spectrum with maxima at particular wavelengths $\left(\lambda_{\mathrm{m}}\right)$ determined by the following formula:

$$
\lambda_{\mathrm{m}}=2 \mathrm{n}_{\text {eff }} \mathrm{d} / \mathrm{m},
$$

where $\mathrm{m}$ is an integer, $\mathrm{d}$ is the layer thickness, and $\mathrm{n}_{\mathrm{eff}}$ is the effective refractive index of the porous layer [6]. When the medium surrounding the porous structure changes (e.g., when flowing a liquid on it), $\mathrm{n}_{\text {eff }}$ changes, and hence, $\lambda_{\mathrm{m}}$ shifts. It is this shift which can be used to perform a sensing assay and detect the presence of the analyte. 


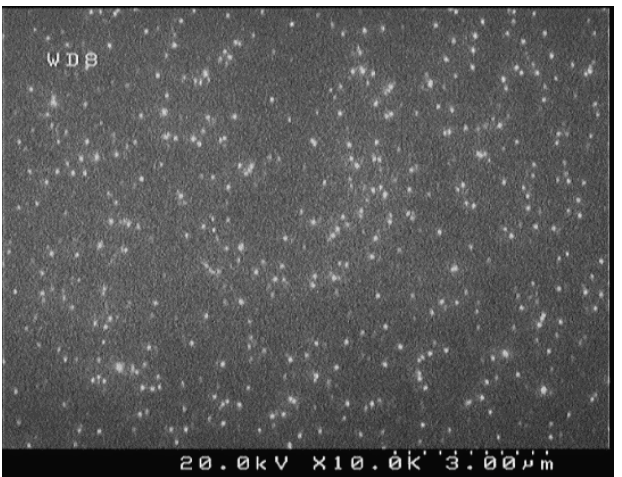

(a)

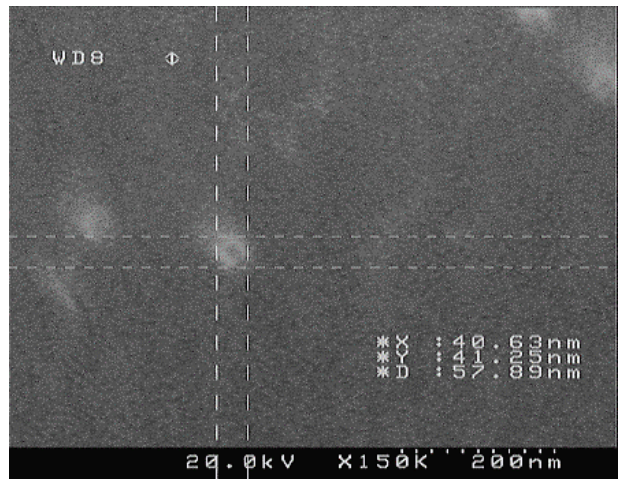

(b)

Figure 1. Surface of a polycarbonate track-etched (PCTE) membrane employed in our assays. Images were taken with a Field-Emission Scanning Electron Microscope (FESEM) microscope. (a) Randomly distributed pores cover the surface. (b) The diameter of the pores are around $30 \mathrm{~nm}$, according to the manufacturer's indication.

To confirm that PCTE membranes could behave like FP interferometers, we characterized their optical response. For that aim, we used a FTIR microscope to perform reflectivity measurements of PCTE membranes surrounded by air in both faces in the NIR region. What we observed was a reflectivity spectrum with several lobes (Figure 2), which is in agreement with the expected spectrum for a FP interferometer.

However, the reflectivity intensity was nearly zero, which could hinder the future sensing assays. Most solutions are prepared with water, and it is well-known that its absorption coefficient in the NIR region is high [9]. If part of the light is absorbed by water and does not arrive to the PCTE membrane, the reflectivity signal will have an even lower intensity. To enhance it, we decided to place the PCTE membrane above a polished silicon wafer, which has flat and higher-intensity reflectivity spectra in the NIR region studied, as it can be seen in Figure 2. This would also provide mechanical stability to the membranes to be used in sensing assays. As we expected, by placing the PCTE membrane on a silicon substrate, the intensity of the reflectivity spectrum was enhanced four-fold (Figure 2).

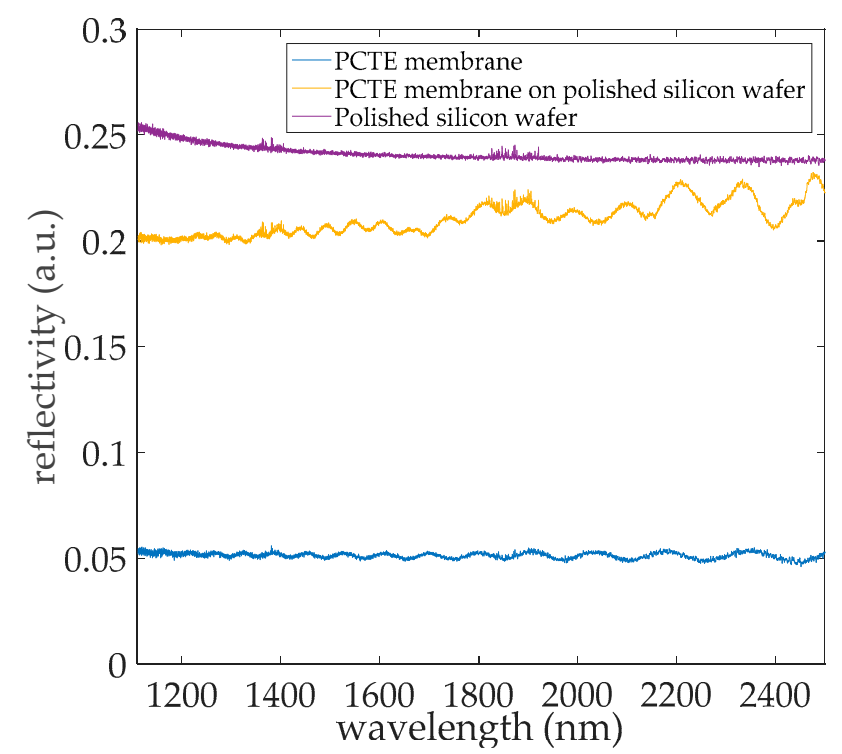

Figure 2. Reflectivity spectra of PCTE membranes with (yellow line) and without (blue line) a silicon substrate below them and a polished silicon wafer (violet). 
In order to covalently bind the PCTE membranes to the silicon substrate, we employed APTES as a crosslinking agent [10]. In this way, we ensured that the membranes would not move while the solutions were flowing in sensing assays.

\subsection{Ethanol Sensing Assays}

Once the optical response of PCTE membranes on a polished silicon wafer was characterized, pure ethanol-sensing assays were performed. For that end, a $10 \mu \mathrm{L}$ drop of ethanol was placed on top of the sample in the area covered by the light beam that arrived from the FTIR. The measurements of the reflectivity spectrum of the sample were taken before the deposition of the drop and during the evaporation process of such a drop to check if a spectral shift could be seen. We expected that the spectrum would shift to longer wavelengths when the air inside the pores was replaced by ethanol, since the effective refractive index of the structure would become higher (see Equation (1)). As ethanol evaporates, the spectrum should return progressively to its initial position, since the pores are being refilled by air and the effective refractive index of the structure decreases.

To check if a shift had occurred, we looked at wavelengths between $2000 \mathrm{~nm}$ and $2500 \mathrm{~nm}$, since here there were the highest-intensity lobes, easing the tracking of the spectral shift. When pores are filled with pure ethanol, we could observe a shift of the spectrum of around $20 \mathrm{~nm}$ (at around 2400 $\mathrm{nm}$ wavelength) toward longer wavelengths. When the ethanol had almost completely evaporated after $3 \mathrm{~min}$, the spectrum went back to the same position as it was initially, when the sample was dry (Figure 3). Differences in reflectivity intensity during the measurement process are due to the layer of liquid created on top of the sample that increased the diffuse component of light reflected by the sample, thus reducing the number of beams arriving to the lenses of the FTIR microscope.

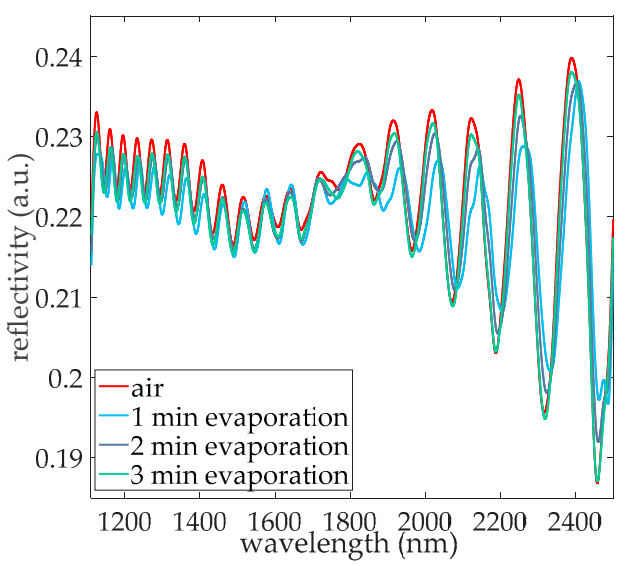

(a)

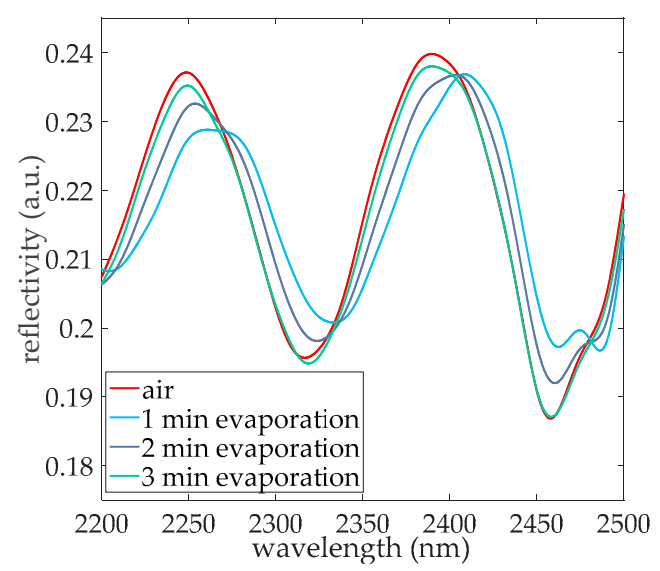

(b)

Figure 3. Optical sensing measurements of pure ethanol employing PCTE membranes. (a) Reflectivity spectrum of the sample between 1110 and $2500 \mathrm{~nm}$ during the evaporation process of pure ethanol. (b) Reflectivity spectrum shifts around $20 \mathrm{~nm}$ at $2400 \mathrm{~nm}$ when pure ethanol filling the pores is observed. When the ethanol evaporates, the spectrum returns to the same position it had been in when it was dry.

Using the same sample, immediately after sensing pure ethanol, we placed another $10 \mu \mathrm{L}$ drop of $50 \%(v / v)$ ethanol aqueous solution. What we then saw was a shift of the spectrum of $16 \mathrm{~nm}$ (at around $2400 \mathrm{~nm}$ wavelength) toward longer wavelengths (Figure 4). In comparison with previous measurements with pure ethanol, this difference in the shift magnitude could be explained by the fact that the refractive index of the $50 \%$ solution is smaller than that of pure ethanol [11]. Hence, the effective refractive index of the medium is smaller when $50 \%$ solution is filling the pores and the spectral shift is smaller. However, we have to take into account that measurements had not been taken in real time, but every minute, and the evaporation rate of pure ethanol and its aqueous solutions are different. Therefore, differences in the spectral shift may be due to the refractive index 
differences of both solutions, but also because the volume of air filling the pores at the same time after depositing the drop is different, because the evaporation rate is different.

If we consider that the evaporation rate did not affect our measurements and, after a minute, the pores filled with pure or $50 \%$ ethanol, we can estimate that the sensitivity of our sensor was around $54 \mathrm{~nm} / \mathrm{RIU}$ (refractive index unit) (50 nm/RIU when sensing $100 \%$ ethanol and $58 \mathrm{~nm} / \mathrm{RIU}$ when sensing $50 \%$ ethanol).

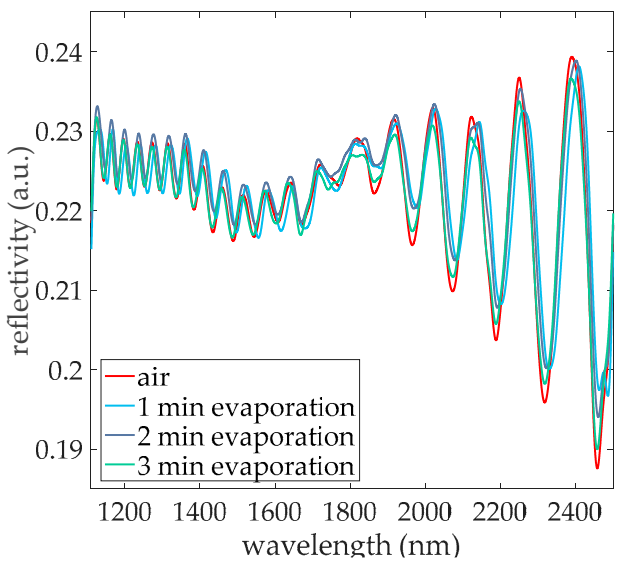

(a)

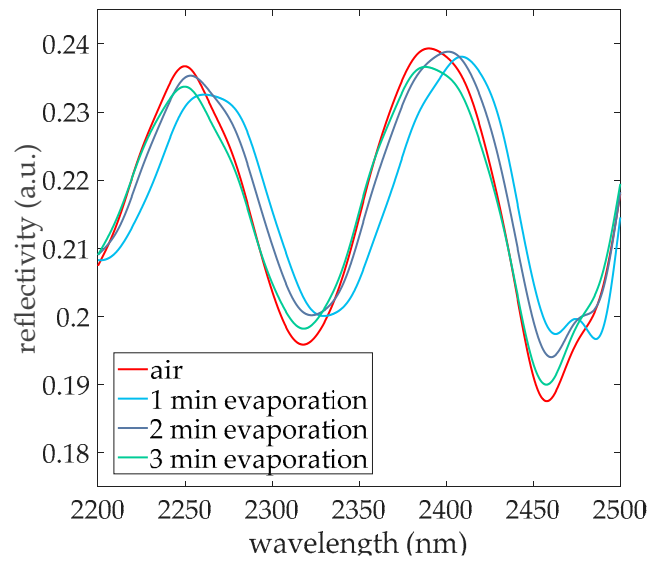

(b)

Figure 4. Optical sensing measurements of a $50 \%(v / v)$ aqueous solution of ethanol employing PCTE membranes. (a) Reflectivity spectrum of the sample between 1110 and $2500 \mathrm{~nm}$ during the evaporation process of $50 \%(v / v)$ ethanol. (b) Reflectivity spectrum shifts around $15 \mathrm{~nm}$ at $2400 \mathrm{~nm}$ when $50 \%(v / v)$ ethanol filling the pores is observed. When the ethanol evaporates, the spectrum returns to the same position it had been in when it was dry.

\section{Discussion}

Our results suggest the utility of PCTE membranes as optical sensors for the detection of ethanol. We demonstrated that these kinds of membranes behave like a FP interferometer when exposed to a light source in the NIR range, in our case. Furthermore, we were able to clearly detect the presence of ethanol in the medium, and could demonstrate the possibility of reusing the same sensor at least twice.

If we compare the sensitivity of our sensors with the sensitivity of PSi FP microcavities, we can see that our PCTE membrane sensors are less sensitive than those ones [5]. However, our sensors are easier to obtain since they are commercially available, which could reduce the cost of the final devices. In order to explore ways of improving our sensitivity, further research must be carried out.

On the other hand, it would be interesting to detect different ethanol concentrations. We could see in our results that the smaller the concentration of ethanol, the smaller the spectral shift. This might indicate that PCTE membranes could allow us to quantify and optically distinguish different concentrations of the analyte. However, it would be incautious to affirm that, since we did not perform real-time measurements and we just tested two different concentrations. Therefore, further assays must be done with different concentrations, and there must be efforts at trying to record measures in a shorter period. Moreover, we would like to explore the possibility of flowing the ethanol, instead of depositing the drop on the sample.

In conclusion, these results open up a new path to explore in relation to creating chemical sensors easily. Furthermore, as polycarbonate can be chemically modified to bind biomolecules to its surface [12], the development of an optical biosensor based on PCTE membranes could also be explored.

\section{Conclusions}

To the best of our knowledge, we have made the first-ever attempt at presenting commercial PCTE membranes as new candidates for developing chemical optical sensors. By employing these membranes, we were able to detect the presence of two different concentrations of ethanol in the 
medium. Furthermore, we could see differences in the spectral shift magnitude in the presence of each concentration. This could indicate a possibility of being able to quantify these new optical sensors. However, as these are only early results, further work should be done to assess that these differences were due to concentration and not to the measurement setup.

This finding could indicate a new way to develop optical sensors which are easier and faster to obtain. PCTE membranes are commercially available, and the etching process required for the fabrication of current porous photonic structures will no longer be necessary. Furthermore, as polycarbonate surfaces can be chemically modified, specific sensors for specific applications could be developed.

Funding: This research was funded by Spanish government through grant TEC2015-63838-C3-1-ROPTONANOSENS and the Universitat Politècnica de València through grants PAID-01-17.

Conflicts of Interest: The authors declare no conflict of interest. The funders had no role in the design of the study; in the collection, analyses, or interpretation of data; in the writing of the manuscript, and in the decision to publish the results.

\section{References}

1. Zhang, Y.; Zhao, Y.; Zhou, T.; Wu, Q. Applications and developments of on-chip biochemical sensors based on optofluidic photonic crystal cavities. Lab Chip 2018, 18, 57-74.

2. Zhang, L.; Zhang, Z.; Wang, Y.; Ye, M.; Fang, W.; Tong, L. Optofluidic Refractive Index Sensor Based on Partial Reflection. Photonic Sens. 2017, 7, 97-104.

3. Ruiz-Tórtola, A.; Prats-Quílez, F.; González-Lucas, D.; Bañuls, M.J;; Maqueira, Á.; Wheeler, G.; Dalmay, T.; Griol; A.; Hurtado, J.; Bohlmann, H.; et al. Experimental study of the evanescent-wave photonic sensors response in presence of molecular beacon conformational changes. J. Biophotonics 2018, doi:10.1002/jbio.201800030.

4. Caroselli, R.; Ponce-Alcántara, S.; Prats-Quilez, F.; Martín-Sánchez, D.; Torrijos-Morán, L.; Griol Barres, A.; Bellieres, L.; Bandarenka, H.; Girel, K.; Bondarenko, V.; García-Rupérez, J. Experimental study of the sensitivity of a porous silicon ring resonator sensor using continous in-flow measurements. Opt. Express 2017, 25, 31651-31659, doi:10.1364/OE.25.031651.

5. Caroselli, R.; Martín-Sánchez, D.; Ponce-Alcántara, S.; Prats-Quilez, F.; Torrijos Morán, L.; García-Rupérez, J. Real-time and in-flow sensing using a high sensitivity porous silicon microcavity-based sensor. Sensors 2017, 17, 2813, doi:10.3390/s1722813.

6. Ponce-Alcántara, S.; Martín-Sánchez, D.; Pérez-Márquez, A.; Maudes, J.; Murillo, N.; García-Rupérez, J. Optical sensors based on polymeric nanofibers layers created by electrospinning. Opt. Mater. Express 2018, 8, 3163-3175.

7. Qiu, H.J.; Li, X.; Xu, H.T.; Zhang, H.J.; Wang, Y. Nanoporous metal as a platform for electrochemical and optical sensing. J. Mater. Chem. C 2014, 2, 9788, doi:10.1039/c4tc01913j.

8. Hasar, U.C.; Ozbek, I.Y.; Cavusoglu, B.; Karacali, T.; Efeoglu, H.; Ertugrul, M.; Barroso, J.J. Characterization of porous silicon Fabry-Pérot optical sensors for reflectivity and transmittivity measurements. IEEE J. Sel. Top. Quantum Electron. 2015, 21, 174-183, doi:10.1109/JSTQE.2014.2365583.

9. Wilson, R.H.; Nadeau, K.P.; Jaworski, F.B.; Tromberg, B.J.; Durkin, A.J. Review of short-wave infrared spectroscopy and imaging methods for biological tissue characterization. J. Biomed. Opt. 2015, 20, 030901.

10. Aran, K.; Lawrence, A.S.; Kamdar, N.; Zahn, J.D. Irreversible, direct bonding of nanoporous polymer membranes to PDMS or glass microdevices. Lab Chip 2010, 10, 548-552, doi:10.1039/b924816a.

11. Lide, D.R. CRC Handbook of Chemistry and Physics; Internet Version 2005; CRC Press: Boca Raton, FL, USA, 2003.

12. Ogonczyk, D.; Jankowski, P.; Garstecki, P. Functionalization of polycarbonate with proteins; open-tubular enzymatic microreactors. Lab Chip 2012, 12, 2743-2748, doi:10.1039/c21c40204a. 